\title{
Special Issue on the NCAA-DoD CARE Consortium Research
}

\author{
Bethany Rowson, Joel D. Stitzel, and Stefan M. Duma \\ Institute for Critical Technology and Applied Sciences, Blacksburg, USA
}

The National Collegiate Athletic Association (NCAA) and the U.S. Department of Defense (DoD) partnered to establish the NCAA-DoD Grand Alliance and the Concussion Assessment, Research, and Education (CARE) Consortium in 2014. The CARE Consortium was established to inform policy decisions concerning concussion and its long-term consequences on health as public concern continued to grow. CARE is now in its second phase and is the largest prospective concussion study ever conducted, with over 41,000 male and female NCAA student-athletes and service academy cadets enrolled. The first phase of CARE focused on defining the clinical and neurobiological natural history of concussion and recovery, along with factors that predict poor outcomes following injury. The second phase was initiated to study the cumulative and long-term effects of concussion and repetitive head impacts.

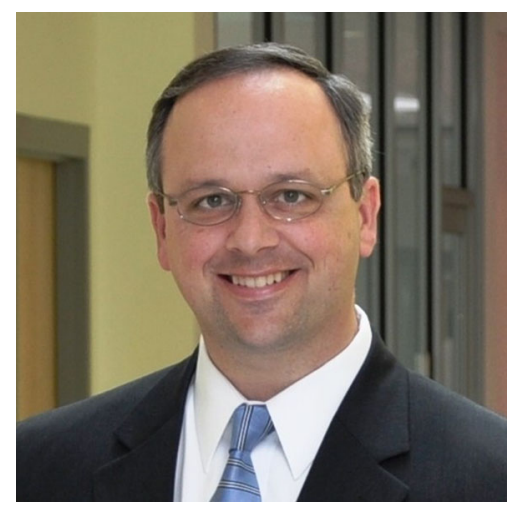

Stefan M. Duma

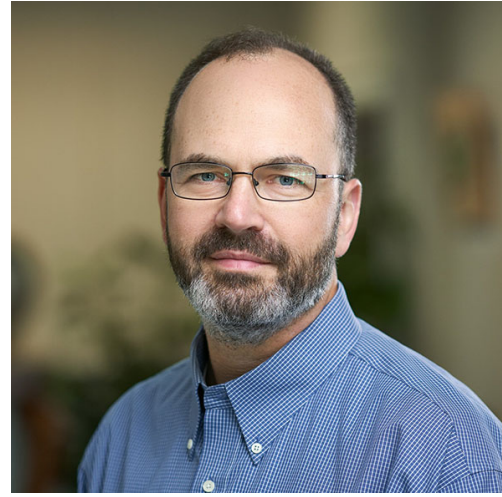

Joel D. Stitzel

The CARE Consortium is one of many initiatives to better understand concussion as an injury and its longterm consequences. As public concern over concussion increases, more research is being devoted to filling in knowledge gaps clinically as well as understanding the mechanics of injuries and how to prevent them. The Annals of Biomedical Engineering (ABME) has published 15 papers related to sports concussions in the last 2 years. The papers covered a wide variety of topics including sensors to measure real-world head impact kinematics, clinical measures of concussion, helmet evaluation, and brain injury modeling. One study presented a novel foam-based technology for measuring real-world head impacts. ${ }^{11}$ Another used existing helmet-mounted sensors to evaluate the correlation between different head injury metrics and concussion risk. ${ }^{13}$ Balance is often used as a component of clinical evaluation of concussion and recovery, but the most common assessments are visually scored by trained personnel, which introduces human error and variance. Due to these limitations, investigators have evaluated different automated methods to detect changes in postural stability. ${ }^{8,12}$ Several studies evaluated the impact performance of existing helmets and newly developed technologies. ${ }^{4,5,10}$ Others looked at helmet testing methods and how they influence helmet evaluation. ${ }^{3,14}$ Finite element modeling has become increasingly important in studying head injury biomechanics since it is difficult to determine the mechanism of injury and relative motion in the brain during impact. Efforts have been made to increase the
Address correspondence to Stefan M. Duma, Institute for Critical Technology and Applied Sciences, Blacksburg, USA. Electronic mail:duma@vt.edu 
complexity and biofidelity of these models for more accurate injury prediction capabilities. ${ }^{1}$ The models have been applied to both real-world and laboratory head impacts to determine strains within the brain that are associated with concussion, and to develop injury metrics that can be used to evaluate the risk of injury in different impact scenarios. , $^{2,9,15}$ In addition to computational models, animal models have also been used to evaluate clinical outcomes of concussion. ${ }^{6}$

Within this special issue devoted to NCAA-DoD CARE Consortium research, the timely papers cover topics related to head impact exposure, head impact sensors, concussion screening tests, and athlete quality of life following a concussion. They build on the recently published concussion research outlined above. Studies looking at head impact exposure quantified differences between exposure in concussed and control athletes and evaluated various factors that affect head impact exposure in athletes. Rowson et al. compared head impact exposure for concussed athletes to matched controls that demonstrated similar traits that may influence concussion tolerance. The concussed athletes experienced more head impacts overall and more highmagnitude impacts than their physically matched controls, putting them at a greater risk for injury. Stemper et al. evaluated repetitive head impact exposure in concussed collegiate football players compared to controls. They found that concussed athletes, in general, had greater head impact exposure both on the day of injury and the time leading up to injury. These results have implications for policies aimed at reducing head impact exposure for athletes. In a separate study, Stemper et al. also evaluated a previously implemented policy meant to reduce head impact exposure by eliminating two-a-day preseason football practices. The results of the study showed that although two-aday practices were eliminated, overall head impact exposure increased because the total number of contact practices was also increased. This study highlights the importance of making data-driven decisions when attempting to reduce head impact exposure.

Two studies evaluated how head impact exposure varies for different players and practice conditions. Campolettano et al. found differences in head impact rate by player position, team, and player ability. Asken et al. identified differences in head impact exposure for different practice drills and player positions. Practice modifications based on these findings could drastically decrease head impact exposure for collegiate football players.

Rich et al. developed a novel mouthpiece sensor for more accurate measurement of head impact kinematics in both non-helmeted and helmeted sports. The sensor was validated in the laboratory and deployed in female youth soccer players on the field. In laboratory tests, data from the mouthpiece sensor were strongly correlated with data from laboratory-grade sensors in an impacted headform. On the field, video-verified impacts were matched with impacts recorded on the mouthpiece, with the mouthpiece sensor demonstrating an overall sensitivity of $69 \%$ and positive predictive value of $80 \%$. These results indicate that this novel sensor could provide accurate on-field measurements of head impact kinematics.

Several cognitive and balance tests are used to assess concussion in athletes and track improvement throughout recovery. Chrisman et al. evaluated the impact of factors that affect reading skill level on baseline performance of a particular screening tool called the King-Devick test. Learning disorders and primary languages other than English were identified as factors that resulted in slower baseline test performance times, indicating that caution should be used with comparisons to normative data for individual athletes. Houston et al. compared results of humanrated vs.sd instrumented balance error scoring system (BESS) scores, a test commonly used to evaluate postural control in athletes following concussion. There was poor agreement between human raters and the instrumented mat used, suggesting that one method should be used for consistency, and the results are likely not comparable.

Weber et al. evaluated global and specific healthrelated quality of life assessments in student-athletes during concussion recovery. The results indicated that even when patients were clinically ready to return to play, some athletes still perceived physical deficits. Additionally, athletes with a history of previous concussions had worse depression scores following injury. The athlete's perception of his or her overall health is an important consideration for concussion recovery and returning to play.

The NCAA-DoD CARE Consortium research has made important advances in understanding concussive injury and recovery. The studies in this special issue further our knowledge base of athlete head impact exposure, and how it varies for individuals and under different practice conditions. New technology to better understand on-field head impact biomechanics and impact exposure was also developed. For concussion recovery, different clinical and quality of life assessments were evaluated to identify limitations and further our understanding of physical and mental recovery. Future CARE Consortium research will expand on these areas as well as study the long-term effects of repetitive head impact exposure and concussions. 


\section{REFERENCES}

${ }^{1}$ Atsumi, N., Y. Nakahira, E. Tanaka, and M. Iwamoto. Human brain modeling with its anatomical structure and realistic material properties for brain injury prediction. Ann. Biomed. Eng. 46:736-748, 2018.

${ }^{2}$ Beckwith, J. G., W. Zhao, S. Ji, A. G. Ajamil, R. P. Bolander, J. J. Chu, T. W. McAllister, J. J. Crisco, S. M. Duma, and $\mathrm{S}$. Rowson. Estimated brain tissue response following impacts associated with and without diagnosed concussion. Ann. Biomed. Eng. 46:819-830, 2018.

${ }^{3}$ Bonin, S. J., J. Luck, C. Bass, J. Gardiner, A. Onar-Thomas, S. S. Asfour, and G. Siegmund. Dynamic response and residual helmet liner crush using cadaver heads and standard headforms. Ann. Biomed. Eng. 45:656-667, 2017. ${ }^{4}$ Clark, J. M., K. Taylor, A. Post, T. B. Hoshizaki, and M. D. Gilchrist. Comparison of ice hockey goaltender helmets for concussion type impacts. Ann. Biomed. Eng. 46:986-1000, 2018. ${ }^{5}$ Demarco, A. L., C. A. Good, D. D. Chimich, J. A. Bakal, and G. P. Siegmund. Age has a minimal effect on the impact performance of field-used bicycle helmets. Ann. Biomed. Eng. 45:1974-1984, 2017.

${ }^{6}$ Esquivel, A. O., S. S. Sherman, C. A. Bir, and S. E. Lemos. The interaction of intramuscular ketorolac (toradol) and concussion in a rat model. Ann. Biomed. Eng. 45:15811588, 2017.

${ }^{7}$ Gabler, L. F., J. R. Crandall, and M. B. Panzer. Development of a metric for predicting brain strain responses using head kinematics. Ann. Biomed. Eng. 46:972-985, 2018.

${ }^{8}$ King, L. A., M. Mancini, P. C. Fino, J. Chesnutt, C. W. Swanson, S. Markwardt, and J. C. Chapman. Sensor-based balance measures outperform modified balance error scoring system in identifying acute concussion. Ann. Biomed. Eng. 45:2135-2145, 2017.

${ }^{9}$ Knowles, B. M., and C. R. Dennison. Predicting cumulative and maximum brain strain measures from HybridIII head kinematics: a combined laboratory study and post hoc regression analysis. Ann. Biomed. Eng. 45:2146-2158, 2017.

${ }^{10}$ Kurt, M., K. Laksari, C. Kuo, G. A. Grant, and D. B. Camarillo. Modeling and optimization of airbag helmets for preventing head injuries in bicycling. Ann. Biomed. Eng. 45:1148-1160, 2017.

${ }^{11}$ Merrell, A. J., W. F. Christensen, M. K. Seeley, A. E. Bowden, and D. T. Fullwood. Nano-composite foam sensor system in football helmets. Ann. Biomed. Eng. 45:27422749, 2017.

${ }^{12}$ Napoli, A., S. M. Glass, C. Tucker, and I. Obeid. The automated assessment of postural stability: balance detection algorithm. Ann. Biomed. Eng. 45:2784-2793, 2017.

${ }^{13}$ O'Connor, K. L., T. Peeters, S. Szymanski, and S. P. Broglio. Individual impact magnitude vs. cumulative magnitude for estimating concussion odds. Ann. Biomed. Eng. 45:1985-1992, 2017.

${ }^{14}$ Trotta, A., D. Zouzias, G. De Bruyne, and A. N. Annaidh. The importance of the scalp in head impact kinematics. Ann. Biomed. Eng. 46:831-840, 2018.

${ }^{15}$ Zhao, W., C. Kuo, L. Wu, D. B. Camarillo, and S. Ji. Performance evaluation of a pre-computed brain response atlas in dummy head impacts. Ann. Biomed. Eng. 45:2437$2450,2017$.

Publisher's Note Springer Nature remains neutral with regard to jurisdictional claims in published maps and institutional affiliations. 